\title{
Étude des effets indésirables lies à l'administration de Sulfadoxine-Pyrimethamine et Amodiaquine lors de la chimio prévention du paludisme saisonnier au Mali
}

\section{Study of Adverse effects related to the administration of Sulfadoxine-Pyrimethamine and Amodioquine during chemoprevention of seasonal Malaria Mali}

\author{
Cisse $\mathrm{B}^{1}$, Diallo $\mathrm{T}^{2,3}$, Traore $\mathrm{D}^{4}$, Denou $\mathrm{A}^{2}$, Coulibaly $\mathrm{SK}^{5}$, DiaraA ${ }^{2}$, Coulibaly $\mathrm{BF}^{2}, \mathrm{Ba} \mathrm{H} \mathrm{S}^{2,6}$, Maiga $\mathrm{A}^{2}, \mathrm{Maiga}^{2}$ \\ ${ }^{1}$ Pharmacie Mosquée Badialan III, Bamako, Mali \\ 2 Département des Sciences du Médicament, Faculté de Pharmacie, USTTB, Mali \\ ${ }^{3}$ Laboratoire National de la Santé du Mali \\ ${ }^{4}$ Point focal de Pharmacovigilance au Mali, Centre National d'appui à la lutte contre les Maladies \\ ${ }^{5}$ Département des Sciences fondamentales, Faculté de Médecine et d'Odonto-Stomatologie, USTTB, Mali \\ ${ }^{6}$ Centre Hospitalier Universitaire du Point G, Bamako, Mali.
}

Auteur correspondant_: Docteur Tidiane Diallo, Maître Assistant en Toxicologie, Département Sciences du Médicament, Faculté de Pharmacie, Université des Sciences, des Techniques et des Technologies de Bamako, Mali.

Email : tidiallo2017@gmail.com

\section{Résumé}

Objectif : L'objectif de notre étude était d'étudier les effets indésirables liés à l'administration de la Sulfadoxine-Pyrimethamine et Amodiaquine lors de la Chimioprévention du paludisme saisonnier (CPS) de 2015 à 2016, dans dix districts sanitaires du Mali.

Population et Méthode : Notre démarche méthodologique était basée sur la collecte des données des effets indésirables à travers une fiche de notification après l'administration des molécules de la Chimio prévention du paludisme saisonnier (Sulfadoxine-Pyrimethamine et Amodiaquine) aux enfants de moins de cinq ans. Les données ont été collectées dans les districts sanitaires de: Nioro du sahel, Nara, Ouelessebougou, Bougouni, Kadiolo, Barouéli, Bla, Ségou, Koro, Tenenkou.

Résultats : Durant notre étude, nous avons enregistré 131 cas d'effets indésirables présentés par 104 enfants. Le district sanitaire de Tenenkou a enregistré plus de cas de notification (50\%), suivi par Nioro du sahel (13\%). Les troubles digestifs étaient les plus représentés soit 83,2\%. L'évolution de l'ensemble des effets indésirables étaient favorables pour tous les enfants.

Conclusion: Le renforcement du système de pharmacovigilance au Mali à travers la formation continue des personnels sanitaires en vue d'une notification continue des effets indésirables pourrait améliorer la prise en charge des effets indésirables liés aux médicaments de la chimio prévention du paludisme saisonnier.

Mots-clés : effets indésirables, Chimio prévention du paludisme saisonnier, Mali.

Abstract

Objective: the occasional preventive treatment defined as the administration of an antimalarial with curative dose in intervals of predefined a time is a very effective and promising strategy of fight against the malaria. One of the important questions concerning the use of this strategy, is the of the medicinal adverse events, it is in this context that we introduced the present study with as objectives general to study the adverse events bound to the administration of SulfadoxinePyrimethamine and Amodiaquine during Chemoprevention of the seasonal malaria.

Population and Method: Our methodological approach was based on the data collection of the EI through an index card of notification after the administration of the molecules of the Chemoprevention of the seasonal malaria to the children at least of 5ans. The data were collected in the sanitary districts of: Nioro of the Sahel, Nara, Ouelessebougou, Bougouni, Kadiolo, Barouéli, Bla, Segou, Koro, and Tenenkou.

Results: During our study, we registered 131 cases of adverse events presented by 104 children. The sanitary district of Tenenkou registered more case of notification $50 \%$, followed by Nioro of the Sahel 13 $\%$. In classifying the EI by clinical sign, we found that the digestive disorders were the most represented or $83.2 \%$. The evolutions of all the EI were favourable.

Conclusion: In the term of this study, can we say that this result does not reflect the reality on the ground with regard to the exhaustiveness of the, he could give some explanation by the sub-notification of the adverse events. So, the strengthening of the system of pharmacovigilance in Mali must be made by the inservice training of the staffs of the health of a continuous notification of the adverse events.

Keywords: adverse events, Chimio prevention of seasonal malaria, Mali. 


\section{INTRODUCTION}

Le paludisme est un problème majeur de santé publique au regard de ses taux de mortalité et morbidité élevés ainsi que ses répercussions socioéconomiques [1]. Il constitue la première cause de consultation dans les structures de santé (40\%). En plus c'est la première cause de décès des enfants de moins de 5 ans et de l'anémie chez les femmes enceintes. En 2015, le nombre de cas de paludisme était de 2.369.245 dont 686.017 cas graves. Chez les enfants de moins de cinq ans, 797.322 cas ont été enregistrés [2]. Dans l'objectif de prévenir l'infection palustre chez les enfants de moins de cinq ans, le Mali a adopté une stratégie basée sur le Traitement Intermittent du Paludisme dénommée: chimio prévention du paludisme saisonnier [3]. Des études faites au Mali, Sénégal, Burkina Faso, Gambie et Ghana, ont prouvé que la Chimio-prévention du Paludisme Saisonnier a entrainé une diminution du nombre de cas de paludisme simple de plus de $80 \%$ et le nombre de cas sévères de plus de 70\% [3]. Au vu de ces résultats encourageants, l'Organisation Mondiale de la Santé a recommandé en mars 2012. Cette stratégie, est de lutter contre le paludisme dans les pays d'Afrique Subsaharienne dont le Mali [4]. Elle consiste en un cycle de traitement complet par la sulfadoxinepyriméthamine (SP) et l'Amodiaquine (AQ) [4]. L'administration de cette association thérapeutique peut provoquer des effets indésirables (EI) plus moins ou grave chez les enfants. Cependant, comme dans toute autre intervention, une surveillance adéquate de la sécurité des médicaments doit être assurée lorsque la CPS est introduit [5]. Un système de pharmacovigilance devient indispensable pour contrôler ces traitements et détecter les effets indésirables mineurs et les effets indésirables graves.. L'Amodiaquine est encore considérée comme un antipaludique mal toléré ayant une toxicité hématologique et hépatique [6]. Les sulfamides sont fréquemment impliqués dans le syndrome de StevensJohnson (SJS) et le syndrome de Lyell. Ces syndromes sont rares, leur fréquence est de l'ordre de 0.01-20 cas/100.000 expositions. Toutefois, elles sont potentiellement mortelles et caractérisées par la destruction brutale de l'épiderme et des muqueuses [7]. La cause la plus fréquente de ces réactions est une « allergie » médicamenteuse, bien établie dans environ $60 \%$ des cas, plausible dans environ $30 \%$. D'autres part, de nombreuses études suggèrent l'intrication des facteurs génétiques (HLAB*5801, HLAB1502) [8], et environnementaux (VIH, Herpes virus) dans la survenue des réactions allergiques aux médicaments [9]. C'est dans ce cadre que nous avons initié cette présente étude avec comme objectif général : d'étudier les effets indésirables liés à l'administration de sulfadoxine-pyrimethamine et amodiaquine lors de la chimio-prévention du paludisme saisonnier, Mali de 2015- 2016, dans dix districts sanitaires du Mali.

\section{MÉTHODOLOGIE}

Il s'agit d'une étude prospective descriptive sur la survenue des EI lors de la chimio-prévention du paludisme saisonnier, Mali 2015- 2016. L'étude s'est déroulée pendant la période de forte transmission du paludisme c'est-à-dire du mois de Juillet 2015 à novembre 2016 dans dix districts sanitaires de cinq régions (tableau I). Etait inclus dans notre étude : tous les enfants qui remplissaient la condition d'éligibilité à la CPS et qui ont manifestés des réactions indésirables après la prise des molécules de la CPS. Les enfants qui ont reçu de médicaments antipaludiques contenant de la SP ou de l'AQ au cours des 30 derniers jours, et les enfants qui sont VIH séropositifs et sous prophylaxie à base de cotrimoxazole ont été exclu de l'étude.

Echantillonnage : c'est un échantillonnage exhaustif par inclusion de tout cas d'EI enregistré lors de la CPS. Les médicaments sont donnés une fois par mois pendant 3-4 mois. Après chaque administration de $\mathrm{SP}+\mathrm{AQ}$ à $\mathrm{J} 1, \mathrm{AQ}$ à $\mathrm{J} 2$ et $\mathrm{J} 3$, l'enfant est protégé pendant 4 semaines. La posologie était fonction de l'âge des enfants :

- Enfants de 3 à 11 mois SP: $250 / 12,5 \mathrm{mg}$ en dose unique AQ: $75 \mathrm{mg}$ pendant 3 jours.

- Enfants de 12 à 59 mois

SP: $500 \mathrm{mg} / 25 \mathrm{mg}$ en dose unique AQ: $150 \mathrm{mg} / \mathrm{j}$ pendant 3 jours

La prise correcte et complète de la CPS doit impérativement être couplée avec une bonne utilisation des moustiquaires imprégnées et des autres méthodes de prévention du paludisme, pour un meilleur résultat.

\section{Collecte des données}

Après chaque passage de la campagne de la CPS, les fiches de notification sont renvoyées au niveau du Centre National d'Appui à la lutte contre la Maladie (CNAM) qui nous a permis d'en avoir accès. En plus des fiches reçues par le circuit habituel, $d$ 'autres fiches ont été scannées puis envoyées par courrier électronique. Enfin nous avons obtenu aussi des informations de notification à travers des appels téléphoniques, vue l'obtention tardives des informations au CNAM. Le principe était d'appeler à partir du CNAM, les agents de la campagne CPS sur le terrain afin de prendre des informations sur la survenue des effets indésirable. Nous avons notifié toutes les informations fournies par les agents de la campagne CPS dans la fiche de notification. Après notification, la fiche était classée avec ceux recueillies sur le terrain. 
La fiche de notification comporte les éléments suivants

- Données sur le patient : âge, sexe, taille, poids, allaitement, adresse.

- Données sur le(s) médicament(s) pris par le patient : désignation, forme, dosage, renseignements sur les caractéristiques du produit, fabricant, $\mathrm{N}^{\circ}$ de lot, date de début et d'arrêt du traitement, posologie, voie d'administration et motif de la prescription

- Données sur les effets indésirables : Description, délai d'apparition après la prise médicamenteuse, évolution à l'arrêt du médicament, traitement correcteur, notion de ré administration avec ou sans rechute si elle a été effectuée, gravité et évolution de l'effet indésirable.

- Données sur le notificateur : nom et prénom, qualification, contact et signature.

\section{Imputabilité}

Pour la réalisation de l'imputabilité nous avons pris les fiches qui ont été suffisamment documentées auxquelles nous avons appliqué l'imputabilité de l'OMS.

Celle-ci est basée sur 3 considérations :

- La relation chronologique entre l'administration du médicament et l'EI.

- La probabilité médicale ou pharmacologique (signes et symptômes, test de laboratoire, données pathologiques, mécanismes).

- La présence ou l'absence d'autres causes.

De ces trois considérations nous avons six résultats possibles :

- Certain/Très probable

- événement clinique ou biologique survenant dans un délai compatible avec la prise de médicament.

- ne pouvant être expliqué par la maladie intercurrente ou par la prise concomitante d'une substance médicamenteuse ou chimique.

- la régression de l'effet doit coïncider avec l'arrêt du médicament (pharmacologique et pathologique)

- événement doit être expliqué par un mécanisme pharmacologique, ou répondre logiquement à une réadministration si nécessaire,

- Probable

- événement clinique ou biologique survenant dans un délai raisonnable après la prise de médicament.

- ne pouvant être expliqué par la maladie intercurrente ou par la prise concomitante d'une substance médicamenteuse ou chimique.

- l'évolution clinique est favorable à l'arrêt du médicament

- l'information sur la réadministration n'est pas nécessaire pour répondre à la définition,

- Possible

- événement clinique ou biologique survenant dans un délai raisonnable après la prise de médicament.

- pouvant être expliqué par la maladie intercurrente ou par la prise concomitante d'une substance médicamenteuse ou chimique.

- l'information se rapportant à l'arrêt du médicament manque ou bien elle est peu claire,

- Improbable

- délai d'apparition de l'événement clinique ou biologique après la prise du médicament rend la relation de cause à effet improbable,

- l'implication de la maladie sous-jacente ou la prise concomitante d'un médicament est à l'origine d'une explication plausible

- Conditionnel/ non classée : événement clinique ou biologique rapporté comme étant un EI du nécessitant plus de d'informations pour être imputé ou bien les données supplémentaire sont à l'étude

- Non évaluable/ non classée : une notification suggérant un effet indésirable qui ne peut pas être imputé car les informations disponibles sont insuffisantes ou contradictoires et qui ne peuvent être ni complétés ni vérifiés.

Saisie et l'analyse des données

Les données étaient enregistrées sur des fiches d'enquête préétablies. La saisie a été faite sur le logiciel Microsoft office Excel et l'analyse statistique sur le logiciel Epi info version 7.1.1.0.

Après la saisie, une vérification et épuration des données ont été faites avant de commencer l'analyse.

Considérations éthiques et déontologiques

L'étude est réalisée dans le respect de l'anonymat et la confidentialité à travers l'obtention d'avis favorable des parents des enfants.

\section{RÉSULTATS}

Durant notre étude, nous avons enregistré 131 cas d'EI présentés par 104 enfants. Les signes cliniques manifestés lors des effets indésirables étaient multiples et variés. Le sex-ratio était en faveur sur sexe féminin 1,1. L'âge moyen des enfants était de 3 ans, la tranche d'âge 12-59 mois était la plus représentée soit $84 \%$ des effectifs. Dans $31 \%$ des cas les enfants étaient 
allaitants. La plus grande couverture est effectuée au quatrième passage dans la région de Koulikoro (664359 enfants) alors que plus de la moitié des EI provenaient de la région de Mopti, le district sanitaire de Tenenkou a rapporté 52 cas de notifications soit $50 \%$ de l'ensemble des notifications (tableau II). Les troubles digestifs étaient majoritairement représentés (109 cas), soit $83,2 \%$ et parmi ces troubles les vomissements et les diarrhées prédominaient avec respectivement 65 et 30 cas soit $49,7 \%$ et $22,9 \%$ (tableau III). Durant notre étude nous avons obtenu plusieurs notifications provenant de types notificateurs de différents grades sanitaires. Plus de la moitié des notifications provenaient des infirmiers (61cas) soit $58,7 \%$ alors que seulement 1 cas était des sages-femmes soit $0,9 \%$, voir figure $\mathrm{N}^{\circ} 1$. Sur les 98 cas de notifications auxquels le mois était renseigné, nous avons enregistré 27 cas de notifications pendant le mois de juillet soit 29,6\%, par contre le d'EI pendant le mois de novembre ne représente que $6,8 \%$ des notifications soit 7 cas (figure $\mathrm{N}^{\circ} 2$ ). Durant notre étude, $91 \%$ des EI sont apparus lors de la première administration c'est à dire le premier jour de la campagne (J1). Sur les 86 cas de notification dont le délai d'apparition était précisé, plus de $55 \%$ ont déclaré des EI survenant dans les 4 heures qui suivent l'administration médicamenteuse. Une prise en charge a été immédiatement mise en évidence, après l'apparition des signes cliniques observés. La plupart des EI étaient corrigés par le metoclopramide et la Sel de Réhydratation Orale qui représentaient chacun $16,1 \%$. Les évènements cliniques qui ont entrainé l'arrêt du traitement CPS représentaient 32\% soit 29 cas. Lors de notre étude nous avons noté une réadministration chez 16 enfants soit $15 \%$ de l'effectif avec une seule réapparition de l'EI après la réadministration. Sur les 104 notifications seulement $4 \%$ sont déclarés graves et ont nécessité une hospitalisation; dont un seul cas a conduit à une prolongation d'hospitalisation par transfert de structure sanitaire. Les 4\% jugé grave n'était pas dû seulement aux médicaments. Il y avait d'autres causes (l'état général de l'enfant, maladie sous-jacente, ...) qui pouvaient expliquer les manifestations en question. Conformément à la méthode d'imputabilité de l'OMS que nous avons utilisée : le résultat était possible dans $72,7 \%$ des effectifs pour les troubles gastro-entériques (tableau V). Cette imputabilité a été effectuée sur 33 $\mathrm{EI}$, car ceux-ci répondaient aux critères d'imputabilités de l'OMS.

\section{DISCUSSION}

Notre étude qui a porté sur les EIM lors de la chimioprévention du paludisme saisonnier au Mali en 2016, nous a permis d'avoir 104 notifications dont 131 EI. Les limites de cette étude concernent :

- la qualité des déclarations, avec le problème des données manquantes concernant les produits incriminés, les autres traitements suivis, les caractéristiques et antécédents du patient, la réaction constatée, la chronologie des évènements, les examens complémentaires réalisés le cas échéant : ce qui a été un frein à la réalisation de l'imputabilité de l'ensemble des déclarations.

- la sous-notification c'est-à-dire les professionnels de santé ne déclarent pas au système de pharmacovigilance tous les effets indésirables qui devraient être signalés, pour diverses raisons parmi lesquelles la non reconnaissance de l'évènement indésirable et la réticence ;

- d'autre part, les fiches remontaient tardivement au niveau de la division en charge de la pharmacovigilance (CNAM); ce qui nous a conduit à procéder à la notification par téléphone (Phone reporting);

Dans notre étude, nous avons trouvé que les enfants de sexe féminin prédominaient avec un ratio 1,1 par contre une étude similaire faite au Niger par Sani Alio en 2016 montre une prédominance en faveur du sexe masculin avec un ratio 1,2 [10]. La région de Mopti a rapporté plus de cas par rapport aux autres régions, soit $53,9 \%$. Ce résultat pourrait s'expliquer par notre présence dans le district sanitaire de Tenenkou. Notre présence a été une source motivation pour les prestataires à notifier tous les cas d'EI rencontrés. Ce résultat montre l'impact de la présence des agents du centre de pharmacovigilance dans les structures sanitaires. Dans plus de 50\%, les notifications provenaient des infirmiers, ce résultat est bien fondamental car au Mali la plupart des centres de santé sont détenus par des infirmiers en plus ils étaient les plus proche des enfants lors de l'administration des médicaments. Nous avions rencontré plus de cas au premier passage soit 52,2\% comparativement aux 2 autres passages. Ce qui pourrait s'expliquer par la forte motivation des parents au début de la campagne à déclarer les EI aux structures sanitaires. Ce résultat est corroboré par des études menées en Gambie par Kalifa Bojang et al qui ont trouvé une proportion égale à $30,5 \%$ [11], Sani Alio au Niger (52,9\%) [10]. Alors que Zongo et al au Burkina n'ont obtenu que $16 \%$ au premier passage de la CPS [12]. Les médicaments utilisés lors de la campagne de la CPS sont susceptibles d'entrainer des EI mineurs mais également majeurs. 
Dans notre étude aucun cas d'atteinte cutanée sévère (type Steven- Johnson ou Lyell) lié au traitement prophylactique n'a été détecté. Aucun EI non attendu (c'est-à-dire qui n'existe pas dans le résumé des caractéristiques du produit) n'a été signalé au cours de cette étude. Les EI, les plus rencontrés étaient mineurs soit $96,9 \%$ et les 4 cas considérés graves par les prestataires sont déclarés guéris sans séquelle avant la fin de notre étude. Ce résultat est confirmé par Sani Alio dans son étude a trouvé 98\% d'EI mineurs [10]. Les troubles digestifs étaient les plus fréquents des EI mineurs, ces troubles représentent $84,4 \%$. Ce résultat est supérieur à l'ensemble des résultats obtenus dans la sous-région. En effet, l'amodiaquine est susceptible d'entrainer des troubles digestifs type, nausée, vomissement, diarrhée. Maiteki-Sebuguzi et al à Uganda en 2008 [13] et Sani Alio au Niger en 2016 [10] ont obtenu respectivement $47 \%$ et $65,9 \%$, tandis que l'équipe de Zongo au Burkina Faso en 2015 CPS [12] et celle de Sockna au Sénégal en 2008 ont trouvé respectivement $20 \%$ et $27 \%$ [14]. Des effets indésirables d'ordre général (fièvre, maux de tête) ont été observés au cours de notre étude. Ces troubles sont renseignés dans la notice d'utilisation et les résumés des caractéristiques de l'amodiaquine. Ces EI mineurs représentaient $7,7 \%$. Ce résultat est similaire à celui trouvé par Sani Alio dans son étude 9,4\% [10] alors que Maiteki Sebuguzi et al en 2008 ont trouvé 28,6 \% [13]. Un EI grave est un évènement qui est à l'origine d'un décès, d'une menace pour la vie du patient au moment de son apparition, d'une nécessité d'hospitalisation ou d'une prolongation d'hospitalisation, de séquelles ou incapacité notable et durable. Certes durant notre étude les 4 cas déclarés, ont entrainé une hospitalisation mais le pronostic vital n'était pas mis en jeu. Durant notre étude, la réadministration a été effectuée chez $15 \%$ de nos enfants, et l'EI n'est réapparu qu'une fois. Dans 32\% des notifications, l'arrêt de traitement a été motivé par l'apparition l'EI, et tous ces cas n'ont pas fait l'objet d'une réadministration. Ces arrêts du traitement dépendaient du jugement des prestataires en fonction des cas. Les traitements correcteurs administrés étaient des traitements symptomatiques et la plupart des molécules utilisées avaient pour indication de restaurer les troubles digestifs qui étaient prédominants (métoclopramide, métamizole, diosmectite, attapulgite mormoiron, métopimazine). La présence de zinc, sel de réhydratation orale et sérum glucosé pourrait s'expliquer par la correction de la déshydratation entrainée par les vomissements et diarrhées. Les cas d'allergies ont été corrigés par la prométhazine et dexamethasone, mais nous n'avons pas d'explication en ce qui concerne l'usage des antibiotiques tels: amoxicilline, métronidazole et cotrimoxazole, buthylhyoscine. La méthode de l'OMS [15] nous a conduits à trois résultats : probable, possible et improbable.

- Probable : les EI survenus dans un délai raisonnable après la prise des médicaments étaient $18,1 \%$, et ne pouvaient être expliqués par une maladie intercurrente ou par la prise concomitante d'une substance médicamenteuse ou chimique. L'évolution clinique de l'EI était favorable à l'arrêt du médicament.

- Possible : les EI survenus dans un délai raisonnable après la prise des médicaments et qui peuvent-être expliqués par une maladie intercurrente ou par la prise concomitante d'une substance médicamenteuse étaient $72,7 \%$, et dont l'information se rapportant à l'arrêt du médicament n'était pas fournie par les notificateurs.

- Improbable : le délai d'apparition de l'événement clinique après la prise des médicaments rend la relation de cause à effet improbable dans $9 \%$ cas.

\section{CONCLUSION}

Cette étude prospective portant sur les EI des médicaments de la CPS, nous a permis de les identifier et aussi de les analyser. Ce traitement prophylactique utilisant la sulfadoxine-pyriméthamine et l'amodiaquine a été bien toléré par les enfants au Mali. La majeure partie des EI rencontrés étaient mineurs 127/131 cas enregistrés. Les EI graves comme le syndrome de Steven Johnson ou le syndrome de Lyell n'ont pas été observés au cours de notre étude. La notification permet d'assurer la sécurité des médicaments et des patients, nous devons encourager les prestataires de santé à plus notifié pour détecter rapidement les effets indésirables inattendus, grave et les effets connus qui augmentent de fréquence. Au Mali, aucun EI grave attribuable à ces médicaments n'a été observé après une surveillance à travers un système de pharmacovigilance basé sur la notification spontanée.

Conflit d'intérêt : Aucun

\section{RÉFÉRENCES}

1. World Health Organisation. World malaria report 2014. Geneva; 2014. Consulté le 20 septembre 2017 sur l'URL :

http://www.who.int/malaria/publications/worl d_malaria_report_2014/en/

2. Daouda Tounga Konaté. Mali : le paludisme représente encore $40 \%$ des motifs de consultations. Consulté le 27 sept. 17 sur 
l'URL :

http://afrique.le360.ma/mali/societe/2016/08/2 4/5082-mali-le-paludisme-represente-encore40-des-motifs-de-consultations-5082

3. Ministère de la santé et de l'hygiène publique. Module de formation du paludisme saisonnier chez les enfants de 3 à 59 mois. Programme national de lutte contre le paludisme, Mai 2015, P1

4. OMS Chimio prévention du paludisme saisonnier par administration de Sulfadoxine Pyriméthamine et d'Amodiaquine aux enfants: guide de terrain [Internet]. Consultable sur l'URL: http://www.who.int/malaria/publications/atoz/ 9789241504737/fr/. Consulté le 12/01/16

5. Organisation Mondiale de la Santé. Recherche sur les approches novatrices pour la surveillance de l'innocuité dans la chimioprévention du paludisme saisonnier. [Internet]. [Cited 2016 Mar 3 7:00] Available from: www.who.int/tdr/grants/CEM-SMCfrench.pdf

6. Organisation Mondiale de la Santé. Assurer la sécurité des interventions de chimioprévention pour la lutte contre les maladies tropicales négligées : conseils pratiques à l'intention des administrateurs de programmes nationaux sur la prévention, le dépistage et la prise en charge des événements indésirables graves. Consulter le 08 janvier 2018 sur l'URL : http://apps.who.int/iris/bitstream/10665/75185 /1/9789242502190_fre.pdf

7. Miller KD, Lobel HO, Satriale RF, Kuritsky JN, Stern R, Campbell CC. Severe cutaneous reactions among American travelers using pyrimethamine-sulfadoxine (Fansidar) for malaria prophylaxis. Am J Trop Med Hyg. 1986 May;35(3):451-8

8. Aimone-Gastin I. Prédispositions génétiques aux réactions d'HS allergiques aux médicaments. Rev Fr Allergol. 2013 Apr;53(3):275-9.

9. Syndrome d'hypersensibilité médicamenteuse avec manifestations systémiques sévères après traitement par minocycline - EM|consulte [Internet]. [C12ited 2016 Apr 14, 14:00]. Available from: http://www.emconsulte.com/rmr/article/146518

10. Sani Alio K. Pharmacovigilance au Niger. Thèse de pharmacie; Faculté de pharmacie, USTTB, Bamako. 2016, P 11

11. Bojang K, Akor F, Bittaye O, Conway D, Bottomley C, Milligan P, et al. A randomised trial to compare the safety, tolerability and efficacy of three drug combinations for intermittent preventive treatment in children. PloS One. 2010; 5(6):e11225

12. Zongo I, Milligan P, Compaore YD, Some AF, Greenwood B, Tarning J, et al. Randomized Noninferiority Trial of Dihydroartemisinin-Piperaquine Compared with Sulfadoxine-Pyrimethamine plus Amodiaquine for Seasonal Malaria Chemoprevention in Burkina Faso. Antimicrob Agents Chemother. 2015; 59 (8):4387-96

13. Maiteki-Sebuguzi C, Jagannathan P, Yau VM, Clark TD, Njama-Meya D, Nzarubara B, et al. Safety and tolerability of combination antimalarial therapies for uncomplicated falciparum malaria in Ugandan children. Malar J. 2008; 7:106.

14. Sokhna C, Cissé B, Bâ EH, Milligan P, Hallett $\mathrm{R}$, Sutherland C, et al. A trial of the efficacy, safety and impact on drug resistance of four drug regimens for seasonal intermittent preventive treatment for malaria in Senegalese children. PloS One. 2008;3(1):e1471

15. Bannwarth B. Critère d'imputabilité des effets indésirables des médicaments. Rev Rhum Monogr. 2010 Apr, 77(2) :173-5 
Tableau I : Répartition des lieux d'étude en fonction des différents passages de la CPS

\begin{tabular}{|c|c|c|c|c|c|c|c|c|c|}
\hline \multirow{2}{*}{ Région } & \multicolumn{4}{|c|}{ Population traitée CPS par passage } & \multirow[t]{2}{*}{ District sanitaire } & \multicolumn{4}{|c|}{ Couverture par passage } \\
\hline & P1 & $\mathrm{P} 2$ & P3 & P4 & & P 1 & $\mathrm{P} 2$ & P3 & P 4 \\
\hline Kayes & 468899 & 48484 & 487592 & 498534 & Nioro du sahel & 50579 & 55221 & 56533 & 56277 \\
\hline \multirow{2}{*}{ Koulikoro } & \multirow{2}{*}{590111} & \multirow{2}{*}{628559} & \multirow{2}{*}{650669} & \multirow{2}{*}{664359} & Nara & 37436 & 51838 & 53673 & 58203 \\
\hline & & & & & Ouelessebougou & 28016 & 29057 & 29457 & 29752 \\
\hline \multirow[b]{2}{*}{ Sikasso } & \multirow{2}{*}{576513} & \multirow{2}{*}{593688} & \multirow{2}{*}{608041} & \multirow{2}{*}{624840} & Bougouni & 61396 & 54265 & 76541 & 84158 \\
\hline & & & & & Kadiolo & 43625 & 47613 & 42516 & 51630 \\
\hline \multirow{3}{*}{ Ségou } & \multirow{3}{*}{437421} & \multirow{3}{*}{81918} & \multirow{3}{*}{521665} & \multirow{3}{*}{532289} & Baroueli & 31013 & 5661 & 37329 & 33883 \\
\hline & & & & & $\mathrm{Bla}$ & 56851 & 6541 & 66706 & 69012 \\
\hline & & & & & Ségou & 8891 & 19672 & 91696 & 94553 \\
\hline \multirow{2}{*}{ Mopti } & \multirow{2}{*}{447308} & \multirow{2}{*}{484115} & \multirow{2}{*}{557275} & \multirow{2}{*}{489327} & Koro & 92702 & 96334 & 96331 & 95663 \\
\hline & & & & & Tenenkou & 37814 & 39457 & 39535 & 49509 \\
\hline
\end{tabular}

Tableau II: Distribution des d'effets indésirables et couverture CPS 2016 .

\begin{tabular}{|c|c|c|c|c|c|c|c|c|c|c|c|c|c|c|c|c|}
\hline \multirow{2}{*}{ Région } & \multicolumn{4}{|c|}{ Population traitée CPS par passage } & \multirow{2}{*}{ District sanitaire } & \multicolumn{4}{|c|}{ Couverture par passage } & \multicolumn{4}{|c|}{ EI par passage } & \multirow[b]{2}{*}{ EI } & \multirow{2}{*}{$\begin{array}{l}\text { Incidence } \\
\text { (région) \%o }\end{array}$} & \multirow{2}{*}{$\begin{array}{l}\text { Incidence } \\
\text { (DS) \% }\end{array}$} \\
\hline & \begin{tabular}{l|l}
$\mathrm{P} 1$ \\
\end{tabular} & \begin{tabular}{|l|} 
P2 \\
\end{tabular} & P3 & $\mathrm{P} 4$ & & \begin{tabular}{|l|l}
$\mathrm{P} 1$ & 1 \\
\end{tabular} & $\mathrm{P} 2$ & P3 & $\mathrm{P} 4$ & $\mathrm{P} 1 \mathrm{I}$ & $\mathrm{P} 21$ & P3 & P 4 & & & \\
\hline Kayes & 468899 & 48484 & 487592 & 498534 & Nioro du sahel & 50579 & 55221 & 56533 & 56277 & 6 & 4 & 1 & 3 & 14 & 0,028 & 0,247 \\
\hline \multirow{2}{*}{ Koulikoro } & \multirow{2}{*}{590111} & \multirow{2}{*}{628559} & \multirow{2}{*}{650669} & \multirow{2}{*}{664359} & Nara & 37436 & 51838 & 53673 & 58203 & 3 & & & & 3 & \multirow{2}{*}{0,024} & 0,051 \\
\hline & & & & & Ouelessebougou & 28016 & 29057 & 29457 & 29752 & 5 & 3 & 4 & 1 & 13 & & 0,436 \\
\hline \multirow[b]{2}{*}{ Sikasso } & \multirow{2}{*}{576513} & \multirow{2}{*}{593688} & \multirow{2}{*}{608041} & \multirow{2}{*}{624840} & Bougouni & 61396 & 54265 & 76541 & 84158 & & & 1 & & 1 & \multirow{2}{*}{0,012} & 0,011 \\
\hline & & & & & Kadiolo & 43625 & 47613 & 42516 & 51630 & 4 & & 1 & 2 & 7 & & 0,135 \\
\hline \multirow{3}{*}{ Ségou } & \multirow{3}{*}{437421} & \multirow{3}{*}{81918} & \multirow{3}{*}{521665} & \multirow{3}{*}{532289} & Baroueli & 31013 & 5661 & 37329 & 33883 & & & 2 & 5 & 7 & \multirow{3}{*}{0,018} & 0,187 \\
\hline & & & & & Bla & 56851 & 6541 & 66706 & 69012 & & & & 2 & 2 & & 0,028 \\
\hline & & & & & Ségou & 8891 & 19672 & 91696 & 94553 & & & & 1 & 1 & & 0,01 \\
\hline \multirow{2}{*}{ Mopti } & \multirow{2}{*}{447308} & \multirow{2}{*}{484115} & \multirow{2}{*}{557275} & \multirow{2}{*}{489327} & Koro & 92702 & 96334 & 96331 & 95663 & & & & 4 & 4 & \multirow{2}{*}{0,1} & 0,041 \\
\hline & & & & & Tenenkou & 37814 & 39457 & 39535 & 49509 & 31 & 3 & 6 & 12 & 52 & & 1,315 \\
\hline
\end{tabular}

Tableau III: signes cliniques; CPS, 2015-2016.

\begin{tabular}{llr}
\hline Troubles & $\begin{array}{l}\text { Manifestations } \\
\text { cliniques }\end{array}$ & $\begin{array}{r}\text { Fréquences } \\
\text { (Pourcentages) }\end{array}$ \\
\hline Digestif $(n=109)$ & Diarrhées & $30(22,9)$ \\
& Douleur Abdominale & $14(10,7)$ \\
\hline
\end{tabular}




\begin{tabular}{llr}
\hline & Vomissements & $65(49,7)$ \\
Troubles généraux $(\mathrm{n}=10)$ & Fièvre & $9(6,9)$ \\
Système Nerveux $(\mathrm{n}=2)$ & Maux de tête & $1(0,8)$ \\
& Somnolence & $2(1,6)$ \\
& & $3(2,3)$ \\
& Démangeaison & $2(1,6)$ \\
Dermo-cutané $(\mathrm{n}=10)$ & Prurit & $2(1,6)$ \\
& Urticaire & $2(1,6)$ \\
& Edème & $1(0,8)$ \\
Total & Allergie & $131(100)$ \\
\hline
\end{tabular}

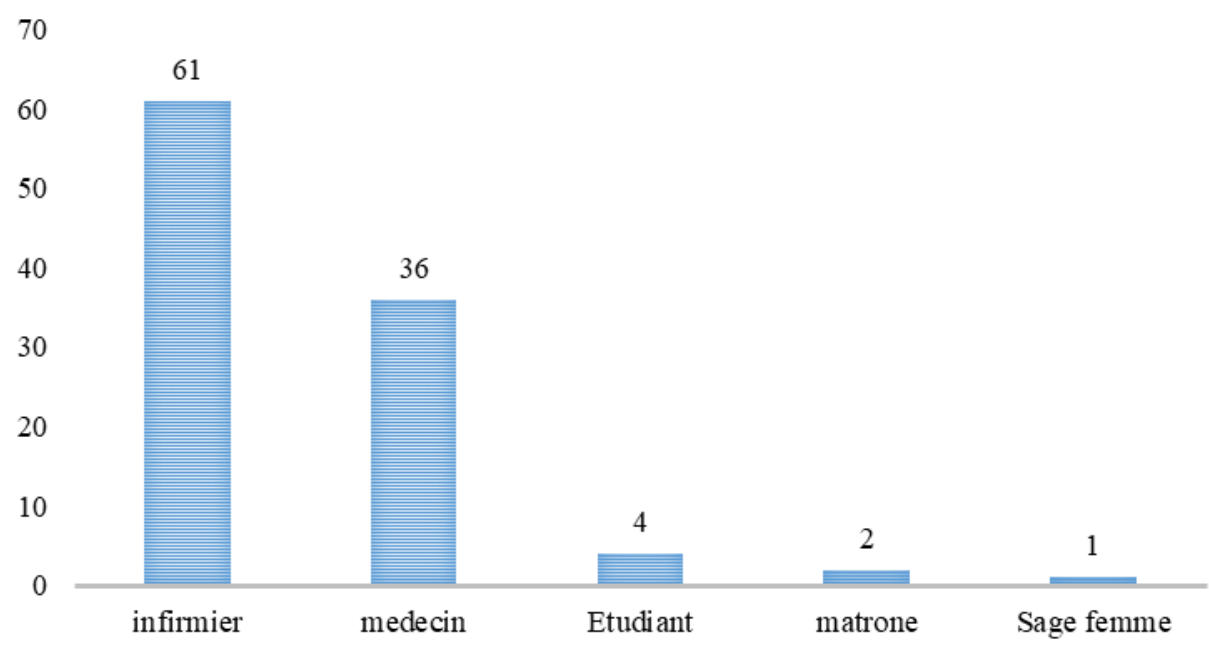

Figure $\mathbf{N}^{\circ} 1$ : Représentation graphique du profil des notificateurs CPS, 2015-2016.

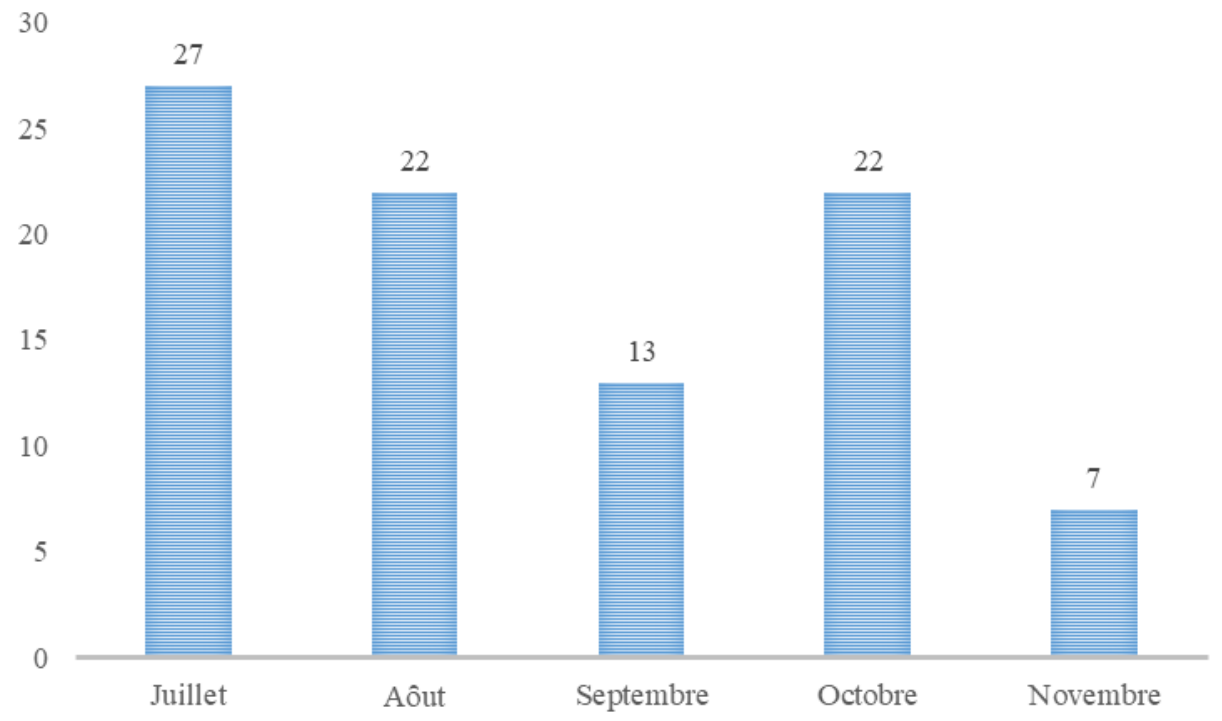

Figure $\mathbf{N}^{\circ} 2$ : représentation des EI en fonction du mois, CPS 2016. 
Tableau IV: Traitements administrés lors de l'apparition des EI

\begin{tabular}{|c|c|c|}
\hline Traitement correcteur & Médicaments & Effectifs (Pourcentage) \\
\hline \multirow{3}{*}{ Antibiotiques $(n=13)$} & Amoxicilline & $1(1,1)$ \\
\hline & Cotrimoxazole & $1(1,2)$ \\
\hline & Métronidazole & $11(12,7)$ \\
\hline \multirow{2}{*}{ Antalgiques $(n=14)$} & Paracétamol & $12(13,8)$ \\
\hline & Métamizole & $2(2,3)$ \\
\hline Antiémétiques (n=17) & Métopimazine & $3(3,4)$ \\
\hline \multirow[b]{2}{*}{ Antidiarrhéiques $(\mathrm{n}=4)$} & Metoclopramide & $14(13,8)$ \\
\hline & $\begin{array}{l}\text { attapulgite de mormoiron } \\
\text { Diosmectite }\end{array}$ & $\begin{array}{l}1(1,1) \\
3(3,4)\end{array}$ \\
\hline \multirow[t]{3}{*}{ Antihistaminiques $(n=8)$} & Prométhazine & $8(9,2)$ \\
\hline & Solution aqueuse & $6(6,9)$ \\
\hline & Buthylhyoscine & $1(1,1)$ \\
\hline \multirow[t]{4}{*}{ Autres $(n=31)$} & Solution de Réhydration Orale & $14(16,1)$ \\
\hline & Zinc & $8(9,2)$ \\
\hline & Dexamethasone & $1(1,1)$ \\
\hline & Sérum glucosé & $1(1,1)$ \\
\hline Total & & $87(100)$ \\
\hline
\end{tabular}

Tableau V: Répartition de 33 cas d'EI selon l'imputabilité de l'OMS

\begin{tabular}{lcr}
\hline Manifestations cliniques & Imputabilité & Pourcentage \\
\hline Troubles gastro-entériques & Possible & 72,7 \\
Troubles Dermatologiques & Probable & 15,1 \\
Troubles Neurologiques & Probable & 3,0 \\
Troubles Généraux & Improbable & 9,0 \\
Total & & $\mathbf{1 0 0 , 0}$ \\
\hline
\end{tabular}

